\title{
Genome-wide analysis of DNA methylation in bronchial washings
}

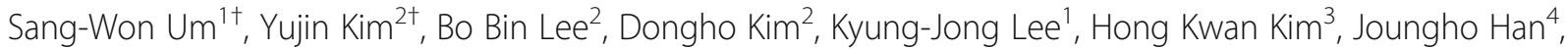
Hojoong Kim', Young Mog Shim ${ }^{3}$ and Duk-Hwan Kim ${ }^{2,5^{*}}$ (D)

\begin{abstract}
Background: The objective of this study was to discover DNA methylation biomarkers for detecting non-small lung cancer (NSCLC) in bronchial washings and understanding the association between DNA methylation and smoking cessation.

Methods: DNA methylation was analyzed in bronchial washing samples from 70 NSCLCs and 53 hospital-based controls using Illumina HumanMethylation450K BeadChip. Methylation levels in these bronchial washings were compared to those in 897 primary lung tissues of The Cancer Genome Atlas (TCGA) data.

Results: Twenty-four CpGs $(p<1.03 \mathrm{E}-07)$ were significantly methylated in bronchial washings from 70 NSCLC patients compared to those from 53 controls. The CpGs also had significant methylation in the TCGA cohort. The 123 participants were divided into a training set $(N=82)$ and a test set $(N=41)$ to build a classification model. Logistic regression model showed the best performance for classification of lung cancer in bronchial washing samples: the sensitivity and specificity of a marker panel consisting of seven CpGs in TFAP2A, TBX15, PHF11, TOX2, PRR15, PDGFRA, and HOXA11 genes were 87.0 and $83.3 \%$ in the test set, respectively. The area under the curve (AUC) was equal to 0.87 (95\% confidence interval $=0.73-0.96, p<0.001$ ). Methylation levels of two CpGs in RUNX3 and MIR196A1 genes were inversely associated with duration of smoking cessation in the controls, but not in NSCLCs, after adjusting for pack-years of smoking.

Conclusions: The present study suggests that NSCLC may be detected by analyzing methylation changes of seven CpGs in bronchial washings. Furthermore, smoking cessation may lead to decreased DNA methylation in nonmalignant bronchial epithelial cells in a gene-specific manner.
\end{abstract}

Keywords: Hypermethylation, Lung cancer, Smoking, Bronchial washing, Epigenome

\section{Background}

Lung cancer is the most common cause of cancer deaths worldwide. Despite recent advances in the diagnosis and treatment for lung cancer, prognosis of patients remains very poor. The overall 5 -year survival rate of lung cancer has improved slightly from 12 to $16 \%$ over the past 30 years [1]. Such poor prognosis is largely due to occult metastatic dissemination of tumor cells, which occurs in

\footnotetext{
* Correspondence: dukhwan.kim@samsung.com

${ }^{\dagger}$ Equal contributors

${ }^{2}$ Department of Molecular Cell Biology, Samsung Biomedical Research Institute, Sungkyunkwan University School of Medicine, Suwon 440-746, South Korea

${ }^{5}$ Samsung Medical Center, Research Institute for Future Medicine, \#50 Ilwon-dong, Kangnam-gu, Professor Rm \#5, Seoul 135-710, South Korea Full list of author information is available at the end of the article
}

more than half of all patients at the time of diagnosis. The majority of patients undergoing curative surgical resection at an early stage have achieved long-term survival. Overall 5-year survival rate for patients with surgically resected stage IA, stage IB, and stage II nonsmall cell lung cancers (NSCLCs) has been reported to be 83,69 , and $48 \%$, respectively [2]. Accordingly, it is imperative to develop efficient diagnostic tools that can identify lung cancer at an early stage so that curative treatment is feasible.

De novo methylation of $\mathrm{CpG}$ islands at the promoter region of tumor suppressor genes is usually associated with transcriptional silencing of a gene. It is one of the most common epigenetic modifications in lung cancer. Aberrant methylation of $\mathrm{CpG}$ loci in bronchial washings

(c) The Author(s). 2018 Open Access This article is distributed under the terms of the Creative Commons Attribution 4.0 International License (http://creativecommons.org/licenses/by/4.0/), which permits unrestricted use, distribution, and 
could become a powerful tool for early diagnosis of lung cancer. To discover aberrant methylation that occur at an early stage of lung cancer, several groups have analyzed methylation statuses of multiple CpG loci in bronchial aspirate or sputum from both lung cancer patients and healthy individuals [3-9]. Epigenetic studies of human cancer have recently shifted from candidate gene analyses toward epigenome-wide analysis with rapid technological advances. However, most studies on bronchial aspirate were restricted to a few candidate $\mathrm{CpG}$ loci with inadequate genome coverage.

Cigarette smoke is a well-known environmental modifier of DNA methylation [10]. It modulates DNA methylation by the following these mechanisms: (i) recruiting DNA methyltransferase 1 (DNMT1) to damage sites during DNA repair; (ii) altering nuclear protein levels and activity of DNA-binding factors such as SP1; or (iii) inhibiting GSK3 $\beta$ function and attenuating DNMT1 degradation [11-13]. Recently, several groups have reported a reversibility of methylation change after smoking cessation in peripheral blood samples. Ambatipudi et al. [14] have reported that methylation levels at smokingrelated $\mathrm{CpG}$ sites are reversible after smoking cessation, although changes in DNA methylation of specific genes can remain for up to 22 years after quitting smoking. Guida et al. [15] have also reported that some methylated CpGs can revert back to levels present in neversmokers after a certain time since smoking cessation.

In this study, we analyzed DNA methylation at the genome level to discover a panel of CpGs for the detection of NSCLC in bronchial washings and investigated the effect of smoking cessation on reversion of methylated DNA to normal levels.

\section{Methods}

\section{Study population}

A total of 123 patients (70 non-small cell lung cancers [NSCLCs] and 53 hospital-based controls with benign lung disease) who were admitted for fiberoptic bronchoscopy or for curative surgical resection at Samsung Medical Center in Seoul, Korea, between March 2010 and November 2015 participated in this study. NSCLC patients underwent surgery as well as bronchoscopy. Controls were recruited from patients with benign lung diseases such as actinomycosis, anthracofibrosis, bronchiolitis, pneumonia, or tuberculosis. Patients with benign lung tumors such as localized organizing pneumonia or hamartoma were excluded from this study. This is because methylation profiling of these diseases is not well known yet which can lead to misclassification. Small-cell lung cancer was also excluded from this study for comparison with The Cancer Genome Atlas (TCGA) methylation profiling. Stage IIIB and IV NSCLCs were excluded because this study was aimed to identify biomarkers for detecting NSCLC at early stages.
Bronchial washings were obtained through bronchoscopy with written informed consent from all participants. The control group received bronchoscopy to confirm the diagnosis. Bronchial washing samples were analyzed cytologically for the presence of malignant cells. All lung cancer patients were diagnosed with earlystage lung cancer which was pathologically proven. They underwent curative resection. Information on sociodemographic characteristics was obtained through an interviewer-administered questionnaire. Individuals who were clinically negative for cancer at the time of bronchoscopy without having abnormal findings on their chest radiograph or chest computerized tomography (CT) were included in the hospital-based control group. This study was approved by the Institutional Review Board (IRB \#: 2010-07-204) of Samsung Medical Center. All cases of NSCLC were classified based on the guideline of tumor-node-metastasis (TNM) staging system introduced by the American Joint Committee on Cancer [16].

\section{Bronchoscopy}

Flexible fiberoptic bronchoscopy (Olympus, Tokyo, Japan) and sample preparation were performed as described previously [4]. Bronchial washing was performed by instilling $10 \mathrm{~mL}$ of sterile normal saline when the bronchoscope (Olympus, Tokyo, Japan) was located at the segmental bronchi of the pulmonary lobe. Bronchial washing samples were mixed with $100 \mathrm{mg}$ of $\mathrm{N}$ acetylcysteine for $10 \mathrm{~min}$ to disrupt disulfide bond in mucoproteins and stored at $-20^{\circ} \mathrm{C}$ until use.

\section{Genome-wide methylation analysis}

Genomic DNA was extracted from bronchial washing specimens using a QIAamp DNA Blood Mini Kit (Qiagen, Valencia, CA, USA) according to the manufacturer's instructions, followed by quality control using a UV spectrophotometer (Pharmacia Biotech, Cambridge, England). Double-stranded DNA in solution was quantitated using PicoGreen $^{\text {Th }}$ double-stranded DNA quantitation kit (Molecular Probes, Eugene, OR, USA) on a SpectraMax Gemini UV spectrometer (Molecular Devices, Sunnyvale, CA, USA). Bisulfite treatment of genomic DNA was performed using Zymo EZ DNA Methylation Kit (Zymo Research, Orange, CA, USA). Genome-wide DNA methylation levels were measured using Infinium HumanMethylation450 BeadChip (Illumina, Inc) according to the manufacturer's instructions. $\beta$ value ranging from 0 (no methylation) to 1 (100\% methylation) was measured as the ratio of signal intensity of methylated alleles to the sum of methylated and unmethylated signal intensity of alleles at each CpG site.

\section{Pyrosequencing}

Methylation levels obtained by $450 \mathrm{~K}$ array were validated by pyrosequencing a cg27364741 locus at the promoter 
region of OTX1 gene using QIAGEN's PyroMark Q24 systems. Biotinylated PCR primer sets for amplification of the locus were purchased from Qiagen (Cat no. PM00616336).

\section{Feature selection for predicting lung cancer}

Preprocessing of $450 \mathrm{~K}$ array data was conducted using wateRmelon [17] and ran on $\mathrm{R}$ programming language. After preprocessing, candidate CpGs for NSCLC prediction were selected in the following order: (i) identifying differentially methylated CpGs; (ii) removing age-related methylation; (iii) performing gene set enrichment analysis; (iv) selecting features; and (v) testing model performance. Gene set enrichment analysis was performed using DAVID (http://david.abcc.ncifcrf.gov/). Annotation clusters with EASE score (a modified Fisher's exact $p$ value) below 1.0E-5 were selected as candidate clusters for model building. Any candidate CpG that was significantly correlated in the same cluster was removed from model building.

\section{Statistical analysis}

$T$ test (or Wilcoxon rank-sum test) and chi-square test (or Fisher's exact test) were used to analyze continuous and categorical variables, respectively. Pearson's (or Spearman's) rank correlation coefficient was used to analyze correlations between two continuous variables. Linear regression analysis was performed to analyze the effect of smoking cessation on DNA methylation after adjusting for potential confounding factors such as packyears of smoking. Multiple logistic regression analysis was performed to discover methylated $\mathrm{CpGs}$ associated with the development of NSCLC after controlling for age, sex, and smoking status. Statistical analysis was conducted using R software (version 3.1.1). Diagnostic performance of the model was measured using a receiver operating characteristic (ROC) curve, which was created with MedCalc statistical software (version 16.8).

\section{Results}

Methylation levels of $450 \mathrm{~K}$ array in bronchial washings were slightly inflated

Assay quality of $450 \mathrm{~K}$ array was tested by comparing measured DNA methylation levels with levels of predefined subsets $(0,33,66$, and $100 \%)$ that were prepared by mixing fully methylated and unmethylated human control DNA (Qiagen, Hilden, Germany). Methylation levels of predefined subsets were similarly reproduced by $450 \mathrm{~K}$ array (Additional file 1: Figure S1A). $\beta$ values from $450 \mathrm{~K}$ array were further confirmed using pyrosequencing (Additional file 1: Figure S1B). A cg27364741 locus at OTX1 gene that was significantly methylated in bronchial washing showed higher methylation in the $450 \mathrm{~K}$ array than that in pyrosequencing, suggesting background signal of the $450 \mathrm{~K}$ array (Additional file 1: Figure S1C).
Data from the $450 \mathrm{~K}$ array were quantile normalized using the wateRmelon $R$ package. Samples were first filtered using the "pfilter" function from the wateRmelon package. Samples having $1 \%$ of $\mathrm{CpG}$ sites with a detection $p$ value $>0.05$, CpG sites containing a beadcount $<3$ in $5 \%$ of samples, and CpG sites with a detection $p$ value $>0.05$ in $1 \%$ of samples were removed. Data preprocessing including background noise removal and type I/type II probes bias correction was conducted using the "dasen" function from the package. A total of $2046(0.42 \%)$ of 485,577 CpGs were filtered out.

\section{Identification of differentially methylated CpGs in bronchial washing}

Clinicopathological characteristics of 53 hospital-based controls and 70 NSCLC patients are described in Additional file 2: Table S1. Average ages of controls and cases were 55 and 64 years, respectively. This difference in age was statistically significant $(p<0.0001)$. The proportion of women was significantly higher in controls than that in cases ( 45 vs. $26 \%, p=0.02$ ). The control group had more never-smokers than the case group ( 55 vs. $29 \%, p=0.007$ ). This required age- and sex-matched controls with absence of significant differences in smoking. However, we could not match controls by age and sex due to low statistical power. Instead, we stratified these data into cases and controls and analyzed age- and smoking-related methylation separately for cases and controls as different cohorts. To identify differentially methylated CpGs in bronchial washings from 70 cases and 53 controls, we transformed the $\beta$ values of methylation level into $\log$ scale $(\log 2[\beta /(1-\beta)])$ because the distribution of $\beta$ values did not follow a normal distribution (ShapiroWilk test, $p<0.05$ ). It was negatively skewed in $450 \mathrm{~K}$ data from bronchial washing samples. Fifty-eight CpGs with $p$ value less than or equal to $1.03 \mathrm{E}-07$ (Bonferroni significance threshold) in two-sided Student's $t$ test were identified from 483,531 CpGs. We selected 31 (Additional file 3: Table S2) out of these 58 CpGs after removing 27 CpGs with a maximum $\beta$ value greater than 0 . 3 in 53 control samples because background signal in the control DNA of $0 \%$ methylation was mostly below $\beta=0.3$. Finally, we applied multiple logistic regression analysis to find $\mathrm{CpGs}$ related to lung cancer after adjusting confounding factors such as age, sex, and the pack-years of smoking. Twenty-four CpGs were found to be significantly methylated in bronchial washing from lung cancer patients than those from healthy individuals. Of the 31 CpGs, the 7 CpGs that were not statistically significant in multiple logistic regression were as follows: SLC15A3 and 6 CpGs (HOXA9, EVX1, HIST1H2BK, EMX1, ITPK, and $P R D M 14$ ) showing age-related methylation (Additional file 4: Figure S2) in 70 NSCLCs and 53 controls. 
Feature selection for lung cancer classification and the performance evaluation of proposed models

The 123 samples were divided into a training set $(N=$ $82)$ and a test set $(N=41)$, and the training set was used to build a classification model. Supervised machine learning algorithms, including support vector machine (SVM), random forest, decision tree, artificial neural network (ANN), logistic regression analysis, and K-nearest neighbor, were used to build models. An optimal subset of CpG features for use in building the classification models was selected from the training set with full $\beta$ values of 485,577 CpGs. The performance of the models was evaluated on the test set using the ROC curve. Among the six algorithms, the logistic regression analysis showed the best classification performance: the sensitivity and specificity of one panel consisting of seven CpGs in TFAP2A, TBX15, PRR15, HOXA11, PDGFRA, TOX2, and PHF11 genes (Fig. 1a) were 87.0 and $83.3 \%$ in the test set, respectively. The area under the receiver operating characteristic (ROC) curve was equal to 0.87 (95\% confidence interval $=073-0.96, p<0.001$; Fig. 1b).

\section{Methylation profiling was different between bronchial washing and surgically resected tumor tissue}

To understand methylation profiling between bronchial washing and surgically resected tumor tissue, we compared the number and $p$ values of CpGs showing statistical significance $(p<1.0 \mathrm{E}-07)$ in 123 bronchial washings and 897 TCGA primary lung tissues (821 primary tumor tissues and 76 normal tissues). All 24 CpGs showing statistical significance in bronchial washings were found to be significantly methylated in the TCGA cohort irrespective of histologic subtypes. The number of statistically significant CpGs was much higher in the TCGA cohort than that in bronchial washings (Fig. 2a). The degree of statistical significance was also lower in bronchial washings compared to that in the TCGA cohort. $p$ values of a CpG showing the strongest significance were $2.2 \mathrm{E}-16$ and $2.1 \mathrm{E}-08$ in the TCGA cohort and bronchial washings, respectively. Over 4000 CpGs were significantly $(p<1.0 \mathrm{E}-07)$ hypermethylated in TCGA tumor tissues compared to normal tissues. Figure 2b-e shows an example of four CpGs in ITGA8, $D L K 1, H T R 1 B$, and RSPO2 genes significantly hypermethylated in the TCGA cohort only. A Gene Ontology (GO) analysis showed that highly methylated genes in the TCGA cohort only were largely involved in the following: (i) positive regulation of transcription from RNA polymerase II promoter, (ii) homophilic cell adhesion via plasma membrane adhesion molecules, (iii) cell-cell signaling, and (iv) G-protein coupled receptor signaling pathway, coupled to cyclic nucleotide second messenger.

\section{Smoking cessation is associated with reduction of methylated CpGs in nonmalignant bronchial epithelial cells} To understand the impact of smoking cessation on DNA methylation in bronchial washing, we first analyzed the association between levels of DNA methylation and three smoking-related variables: age at which smoking began, pack-years of cigarette smoking, and smoking status. The age at which smoking began was not associated with DNA methylation in bronchial washing (data not shown). However, aberrant methylation of nine CpGs in RUNX3, MIR196A1, HOXA11, OTP, GATA4, PTPRU, SLC15A3, ZIC1, and TFAP2B of 24 genes were found to be significantly associated with pack-years of smoking in controls (Additional file 5: Table S3). In addition, methylation patterns of these nine CpGs were different according to smoking status (Fig. 3). Methylation levels ( $\beta$ values) of seven of these nine CpGs were not associated with duration after smoking cessation in NSCLC or control group
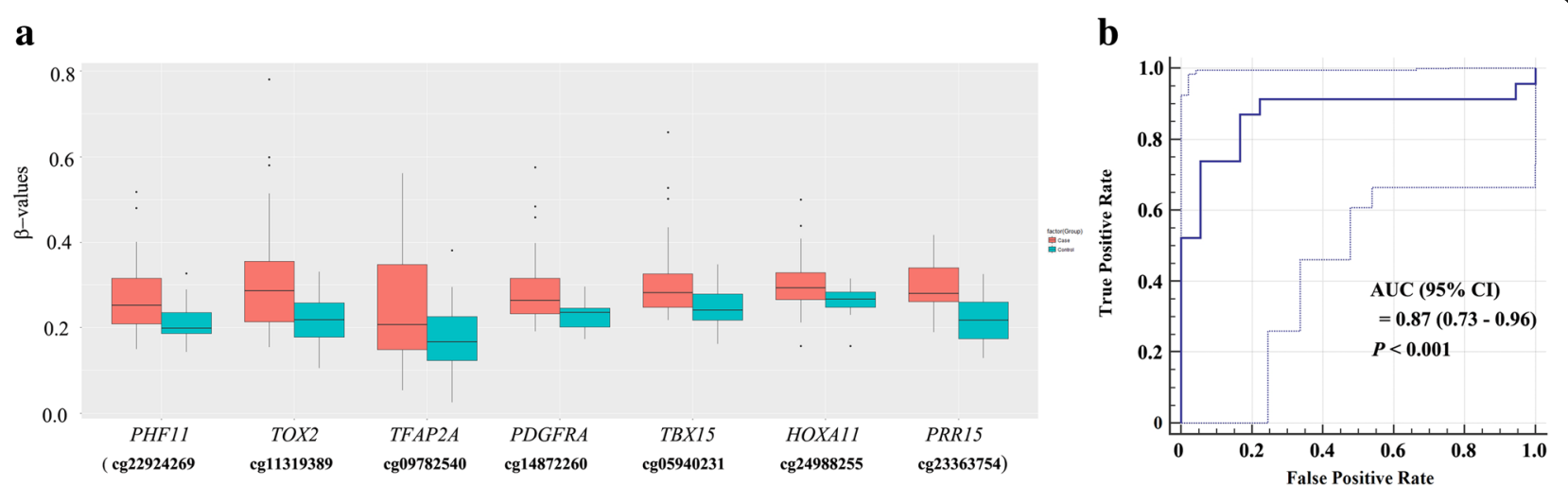

Fig. 1 Prediction performance of a panel of seven CpGs. a Methylation levels of CpGs on seven genes were compared between 45 NSCLCs and 37 controls in a training set. All CpGs showed significant difference $(p<0.05)$ in $\beta$ values between the two groups. $\mathbf{b}$ The prediction performance of the seven-CpG panel was evaluated in 41 test samples. The area under curve of the receiver operating characteristic curve in predicting NSCLC using the panel was 0.87 (95\% confidence interval: 0.73-0.96; $p<0.001)$. The $X$ - and $Y$-axes indicate false positive rate $(1-$ specificity) and true positive rate (sensitivity), respectively 

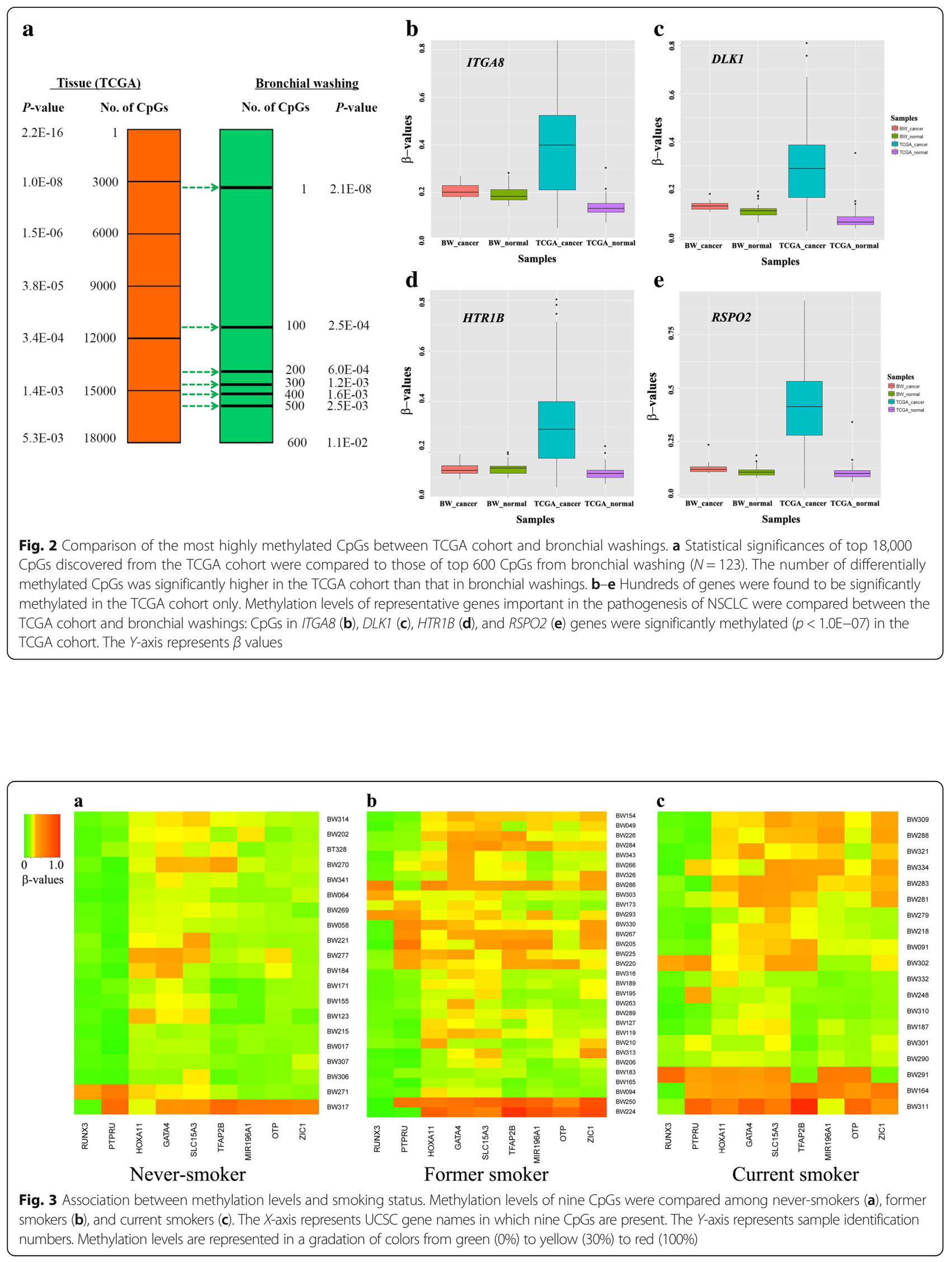
(data not shown). However, an inverse association was found between duration of smoking cessation and methylation levels of two CpGs in RUNX3 and MIR196A1 genes in the control group (Spearman's correlation analysis; Fig. 4a), but not in NSCLC patients (Fig. 4b), suggesting that a decrease in methylation level by smoking cessation might not occur after cancer has formed. Multiple linear regression analysis in the control group showed an inverse relationship of methylation levels of CpG loci on RUNX3 ( $p=0.02)$ and MIR196A1 genes $(p=0.006)$ with duration of smoking cessation, after adjusting for pack-years of smoking (Table 1).

\section{Discussion}

Bronchoscopic examination for lung cancer diagnosis has been developed to overcome the limitation of sputum cytology. Several groups have reported aberrant methylation of $\mathrm{CpG}$ islands at the promoter region of tumor suppressor genes including p16, RASSF1A, $R A R B 2$, and $A P C$ in bronchial aspirate or sputum [3-9].
However, aberrant methylation does not indicate that methylation is tumor-specific since aberrant methylation can occur in bronchial epithelial cells due to aging. Agerelated methylation has been reported for several genes, including $E R, N 33, M Y O D$, thrombospondin-4, and IGF2 [18-22]. Epigenome-wide analysis has also shown changes in DNA methylation with age. Florath et al. [23] have analyzed DNA methylation using $450 \mathrm{~K}$ array in blood DNA of 965 participants aged between 50 and 75 years and identified 65 novel CpG sites associated with aging. Bell et al. [24] have also reported age-related methylation changes in a healthy aging population at genome-wide level. Six of $31 \mathrm{CpGs}$ with significant methylation in the univariate analysis of the present study showed age-related methylation in 53 controls and 70 NSCLCs (Additional file 4: Figure S2).

The number of CpGs showing statistical significance (Bonferroni-corrected $p<0.05$ ) was less in bronchial washings compared to that in the TCGA cohort $[25,26]$ and bronchial biopsy [27]. CpGs of well-known genes such as $R A R \beta 2, C D H 13$, and $p 16$ were not included in

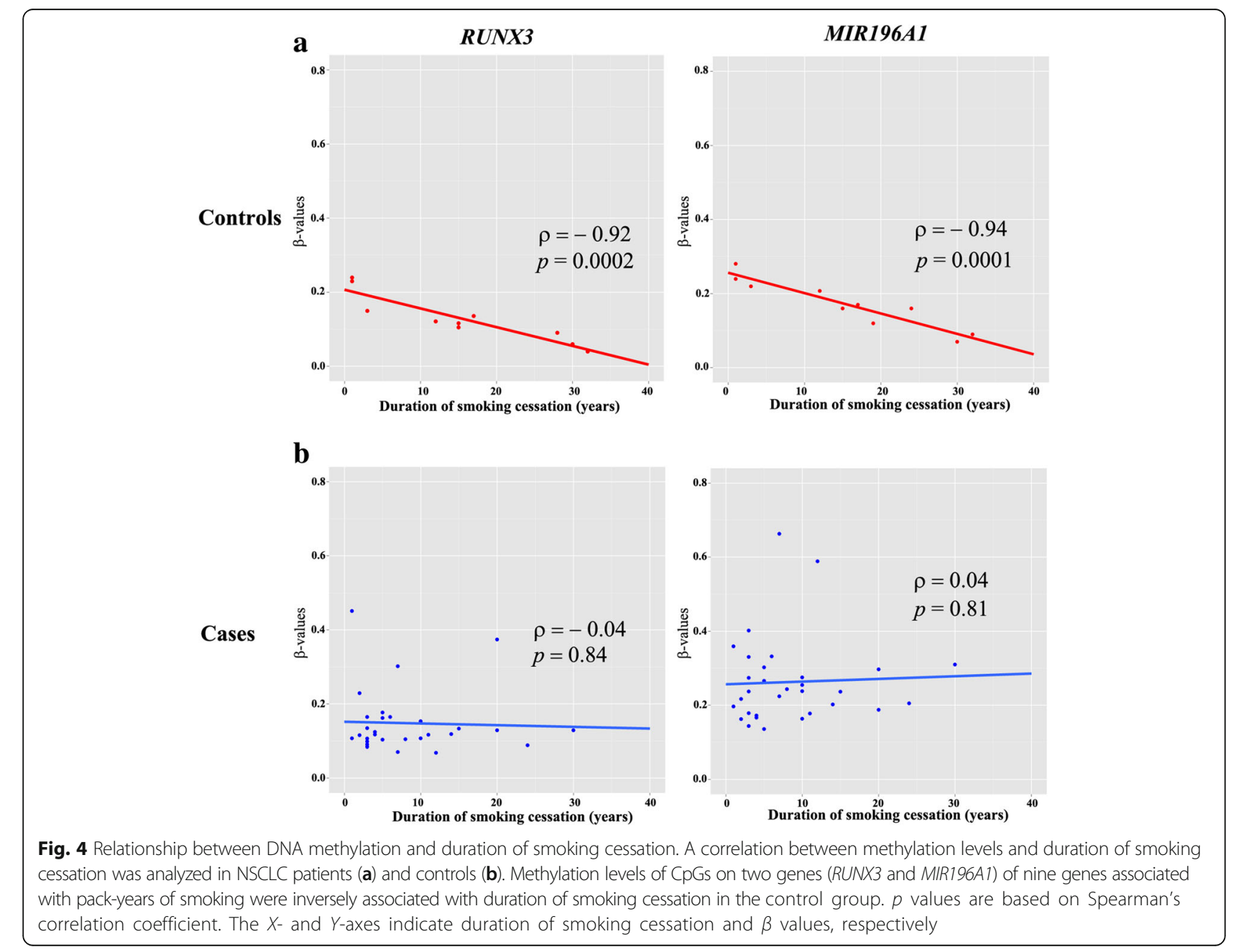


Table 1 Multivariate regression analysis (adjusted for pack-years of smoking) for the association between DNA methylation and smoking cessation in former smokers

\begin{tabular}{llllll}
\hline Parameter & \multicolumn{2}{l}{ Cases $(N=31)$} & & \multicolumn{2}{l}{ Controls $(N=10)$} \\
\cline { 2 - 3 } \cline { 6 - 7 } & $t$ value $^{a}$ & $\operatorname{Pr}>|t|$ & & $t$ value & $\operatorname{Pr}>|t|$ \\
\hline (1) RUNX3 & -0.32 & 0.75 & & -3.30 & 0.02 \\
(2) MIR196A1 & -0.30 & 0.76 & & -4.15 & 0.006 \\
\hline
\end{tabular}

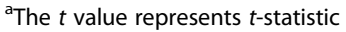

our final CpG list since they did not meet the Bonferroni significance threshold. The small number of CpGs with statistically significant results in bronchial washings compared to the TCGA cohort might be due to contamination of normal bronchial epithelial cells during bronchial washing. Another possibility is that invasive lung cancer cells might undergo more molecular changes than bronchial epithelial cells. In addition to $24 \mathrm{CpGs}$, hundreds of CpGs were found to be significantly hypermethylated in the TCGA cohort, supporting the concept of field cancerization [28]. Widespread cellular and molecular changes during transformation of a precancerous lesion into a cancerous lesion in airway epithelia exposed to carcinogens might also occur in lung tumor tissues. Abnormal methylation found in the TCGA cohort does not mean it has diagnostic value as it can be tumor related, inflammatory cell related, or stromal response related. However, 24 CpGs that were significantly methylated in our bronchial washing samples also showed significant methylation in the TCGA cohort, suggesting that abnormal methylation found in bronchial washing might reflect some methylation changes found in tumor tissue.

A positive relationship between aberrant methylation of genes and exposure to tobacco smoke has been reported by a few groups. For example, aberrant methylation of $p 16$ is associated with smoking duration in primary NSCLC [29]. It was induced by tobacco-specific carcinogen, 4-methylnitrosamino-1-(3-pyridyl)-1-butanone (NNK), in lung of Fischer 344 rats [30]. Aberrant methylation of $p 16, R A R \beta 2, C D H 13$, and $R A S S F 1 A$ genes has also been found in specimens of bronchial epithelial cells from cancer-free heavy smokers [31]. BuroAuriemma et al. [32] have also reported that cigarette smoking can alter methylation patterns in small airway epithelium. In this study, aberrant methylation of nine CpGs was found to be significantly associated with packyears of smoking in bronchial washings of the control group. Among these genes, aberrant methylation of CpGs on GATA4, EMX1, and RUNX3 genes is known to be associated with smoke exposure [33-36]. Wood smoke exposure is associated with lower percent predicted FEV1 of COPD patients in presence of aberrantly methylated GATA4 [33]. Methylation levels of EMX1 are associated with pack-years of smoking in gastric mucosa of healthy individuals [34]. Positive relationship of RUNX3 methylation to smoking has also been observed in DNA from blood leukocytes and placenta [35, 36].

Smoking cessation is a priority for preventing lung cancer. Epigenetic changes after smoking cessation have recently been identified by several groups. Several distinct CpG sites showing decreased methylation with increasing time after smoking cessation in peripheral blood lymphocytes of participants from KORA S3 survey [37] and the European Prospective Investigation into Cancer and Nutrition (EPIC) cohort [14, 15] have been reported, suggesting that a reduction of DNA methylation levels after smoking cessation may occur in a CpG site-specific manner. In this study, the impact of smoking cessation on aberrant methylation of CpG islands was analyzed in bronchial washings of former smokers. Of nine CpGs that were related to pack-years of smoking, two CpGs in RUNX3 and MIR196A1 genes showed an inverse relationship between the duration of smoking cessation and methylation levels of CpGs in the control group after adjusting for pack-years of smoking. Aberrant methylation of MIR196A1 has not yet been reported in human cancer. However, RUNX3 has been reported to be methylated in lung cancer [38, 39]. Runt-related transcription factor 3 (RUNX3) is known to function as a tumor suppressor. It also plays an important role in the regulation of cell proliferation, apoptosis, angiogenesis, cell adhesion, and invasion [40-42]. Reduction in DNA methylation levels after smoking cessation was not found in bronchial washings from lung cancer patients. Accordingly, smokers are encouraged to quit smoking prior to malignant transformation of bronchial epithelial cells.

Not many genes were found to be hypermethylated in bronchial washing in this study. This might be because only well-known genes found in lung cancer tissues were analyzed by candidate gene approach in bronchial washing fluids. Aberrant methylation of DRD5, PHF11, and TPM1 genes discovered in this study has not been known in NSCLC up to date. CpG islands in HOXA11 have been found to be hypermethylated in invasive NSCLC [43] and adenocarcinoma in situ (AIS) [44]. PDGFRA has been found to be significantly methylated in patients with NSCLC $[25,26]$. GDNF and TFAP2A have been reported to be highly methylated in squamous cell carcinoma of the lung [45] and in adenocarcinoma [46], respectively. TOX high mobility group box family member 2 (TOX2) is not methylated in normal lung cancer cells. However, it is methylated in approximately $28 \%$ of lung cancer tissues [47].

This study was limited by several factors. First, this study was conducted in a small number of former smokers. The classification performance of a $\mathrm{CpG}$ panel from bronchial washing and the effect of smoking cessation on DNA methylation should be demonstrated in a 
large cohort in the future. Second, we did not measure the relative proportion of many other cell types (macrophages, lymphocytes, neutrophils) or total cell count in bronchial washing specimens. Therefore, it was unclear how much non-bronchial epithelial cell populations contributed to the analysis of methylation. Third, all cases were early-stage lung cancer and the size of some cancer tissues was small. Thus, we could not analyze methylation patterns of cancer tissues. Methylation patterns of tumor samples and bronchial washing samples should be compared in the same patient. Fourth, the potential impact of tumor heterogeneity and differences between methylation in invasive margin vs. tumor center need to be explored as explanations for the difference seen between bronchial washings and the TCGA cohort. Finally, the sensitivity and specificity of the CpG panel might be inflated because its prediction value was obtained from the same set of patients. Accordingly, an independent set of patients should be used in the future to determine their true values.

\section{Conclusions}

The present study suggests that methylation analysis in bronchial washing may be helpful for detecting NSCLC. Furthermore, smoking cessation in patients with benign lung diseases may result in decreased DNA methylation in a gene-specific manner.

\section{Additional files}

Additional file 1: Figure S1. Validation of 450K array. (A) A quality of $450 \mathrm{~K}$ array was first checked by analyzing measured values for predefined subsets of methylation levels (0,33,66, and 100\%). 2-D scatter plots were produced using plotColorBias2D in the Lumi package. The plotColorBias2D function separately plots methylated (green) and unmethylated (red) probe intensities in a 2-D scatter plot, and shows the interrogated $\mathrm{CpG}$ sites in red and green dots based on their color channels. (B) Methylation levels obtained by the $450 \mathrm{~K}$ array were further validated using pyrosequencing. The sequencing output shows methylation levels at a cg27364741 locus at a promoter of OTX1 gene in cancer (top) and control sample (bottom). (C) Methylation levels at the cg27364741 locus were compared between $\beta$ values from $450 \mathrm{~K}$ array $(Y$-axis) and pyrosequencing $(X$-axis). Pyrosequencing was performed in 12 bronchial washing samples. Methylation levels at the cg27364741 locus were found to be higher in 450K array than in pyrosequencing. (TIF $25467 \mathrm{~kb}$ )

Additional file 2: Table S1. Clinicopathological characteristics $(N=123)$ (DOCX $17 \mathrm{~kb}$ )

Additional file 3: Table S2. Significantly methylated CpGs in bronchial washings of lung cancer. (DOCX $23 \mathrm{~kb}$ )

Additional file 4: Figure S2. Correlation coefficients for 6 CpGs showing positive correlation between patient's age and methylation. The relationship between patient's age and methylation levels. A correlation between methylation levels of EMX1 (A), ITPKA (B), EVX1 (C), HIST1H2BK (D), HOXA9 (F), and PRDM14 (FG) genes and patient's age was analyzed in 70 NSCLC patients and 53 controls separately. $p$ values were based on Spearman's rank correlation coefficient. The $X$ - and $Y$-axes indicate patient's age and $\beta$ values, respectively. (TIF $2963 \mathrm{~kb}$ )

Additional file 5: Table S3. The correlation between pack-years of smoking and DNA methylation in 53 control groups. (DOCX $15 \mathrm{~kb}$ )

\section{Abbreviations}

AUC: Area under the curve; DNMT: DNA methyltransferase; GO: Gene Ontology; NSCLC: Non-small cell lung cancer; TCGA: The Cancer Genome Atlas;

TNM: Tumor-node-metastasis

\section{Acknowledgements}

The authors wish to thank Eunkyung Kim and Jin-Hee Lee for data collection and management, and Hoon Suh for sample collection.

\section{Funding}

This work was supported by a grant (H18C1098) from the Korea Health Technology R\&D Project through the Korea Health Industry Development Institute (KHIDI), funded by the Ministry of Health \& Welfare, Republic of Korea.

\section{Authors' contributions}

S-WU, YK, and D-HK participated in the design of the study, analyzed all data and wrote the manuscript. Pathologic diagnosis was made by JH. Bronchoscopy was performed by S-WU, K-JL, and HK. YK, BBL, and DK conducted genome-wide methylation analysis and pyrosequencing. Surgical resection of lung cancer patients was performed by HKK and YMS. All authors read and approved the final manuscript.

\section{Ethics approval and consent to participate}

All patient samples were obtained after receiving written informed consent from all participants. This study was approved by the Institutional Review Board of Samsung Medical Center (IRB \#: 2010-07-204).

\section{Competing interests}

The authors declare that they have no competing interests.

\section{Publisher's Note}

Springer Nature remains neutral with regard to jurisdictional claims in published maps and institutional affiliations.

\section{Author details}

'Department of Internal Medicine, Samsung Medical Center, Sungkyunkwan University School of Medicine, Seoul 135-710, South Korea. ${ }^{2}$ Department of Molecular Cell Biology, Samsung Biomedical Research Institute, Sungkyunkwan University School of Medicine, Suwon 440-746, South Korea. ${ }^{3}$ Department of Thoracic and Cardiovascular Surgery, Samsung Medical Center, Sungkyunkwan University School of Medicine, Seoul 135-710, South Korea. ${ }^{4}$ Department of Pathology, Samsung Medical Center, Sungkyunkwan University School of Medicine, Seoul 135-710, South Korea. ${ }^{5}$ Samsung Medical Center, Research Institute for Future Medicine, \#50 Ilwon-dong, Kangnam-gu, Professor Rm \#5, Seoul 135-710, South Korea.

Received: 19 December 2017 Accepted: 9 May 2018

Published online: 18 May 2018

\section{References}

1. Siegel R, Naishadham D, Jemal A. Cancer statistics, 2012. CA Cancer J Clin. 2012;62:10-29.

2. Yamamoto K, Ohsumi A, Kojima F, Imanishi N, Matsuoka K, Ueda M, et al. Long-term survival after video-assisted thoracic surgery lobectomy for primary lung cancer. Ann Thorac Surg. 2010;89:353-9.

3. Ahrendt SA, Chow JT, Xu LH, Yang SC, Eisenberger CF, Esteller M, et al. Molecular detection of tumor cells in bronchoalveolar lavage fluid from patients with early stage lung cancer. J Natl Cancer Inst. 1999;91:332-9.

4. Kim H, Kwon YM, Kim JS, Lee H, Park JH, Shim YM, et al. Tumor-specific methylation in bronchial lavage for the early detection of non-small-cell lung cancer. J Clin Oncol. 2004;22:2363-70.

5. Kersting M, Friedl C, Kraus A, Behn M, Pankow W, Schuermann M. Differential frequencies of p16(INK4a) promoter hypermethylation, p53 mutation, and K-ras mutation in exfoliative material mark the development of lung cancer in symptomatic chronic smokers. J Clin Oncol. 2000;18:3221-9.

6. Grote HJ, Schmiemann V, Geddert H, Rohr UP, Kappes R, Gabbert HE, et al. Aberrant promoter methylation of p16(INK4a), RARB2 and SEMA3B in bronchial aspirates from patients with suspected lung cancer. Int J Cancer. 2005;116:720-5.

7. Nikolaidis G, Raji OY, Markopoulou S, Gosney JR, Bryan J, Warburton C, et al. DNA methylation biomarkers offer improved diagnostic efficiency in lung cancer. Cancer Res. 2012;72:5692-701. 
8. Belinsky SA, Palmisano WA, Gilliland FD, Crooks LA, Divine KK, Winters SA, et al. Aberrant promoter methylation in bronchial epithelium and sputum from current and former smokers. Cancer Res. 2002;62:2370-7.

9. Millares L, Rosell A, Setó L, Sanz J, Andreo F, Monsó E. Variability in the measurement of the methylation status of lung cancer-related genes in bronchial secretions. Oncol Rep. 2014;32:1435-40.

10. Breitling LP, Yang R, Korn B, Burwinkel B, Brenner H. Tobacco-smoking-related differential DNA methylation: 27K discovery and replication. Am J Hum Genet. 2011:88:450-7.

11. Mortusewicz O, Schermelleh L, Walter J, Cardoso MC, Leonhardt H. Recruitment of DNA methyltransferase I to DNA repair sites. Proc Natl Acad Sci U S A. 2005; 102:8905-9.

12. Di YP, Zhao J, Harper R. Cigarette smoke induces MUC5AC protein expression through the activation of Sp1. J Biol Chem. 2012;287:27948-58.

13. Lin RK, Hsieh YS, Lin P, Hsu HS, Chen CY, Tang YA, et al. The tobacco-specific carcinogen NNK induces DNA methyltransferase 1 accumulation and tumor suppressor gene hypermethylation in mice and lung cancer patients. J Clin Invest. 2010;120:521-32.

14. Ambatipudi S, Cuenin C, Hernandez-Vargas H, Ghantous A, Le Calvez-Kelm F, Kaaks $R$, et al. Tobacco smoking-associated genome-wide DNA methylation changes in the EPIC study. Epigenomics. 2016;8:599-618.

15. Guida F, Sandanger TM, Castagné R, Campanella G, Polidoro S, Palli D, et al. Dynamics of smoking-induced genome-wide methylation changes with time since smoking cessation. Hum Mol Genet. 2015;24:2349-59.

16. Edge SB, Byrd DR, Compton CC, Fritz AG, Greene FL, Troth A. American Joint Committee on Cancer. In: AJCC Cancer staging manual. 7th ed. New York: Springer; 2010. p. 253-70.

17. Pidsley R, Wong CCY, Volta M, Lunnon K, Mill J, Schalkwyk LC. A data-driven approach to preprocessing Illumina 450K methylation array data. BMC Genomics. 2013:14:293.

18. Issa JP, Ottaviano YL, Celano P, Hamilton SR, Davidson NE, Baylin SB. Methylation of the oestrogen receptor CpG island links ageing and neoplasia in human colon. Nat Genet. 1994;7:536-40.

19. Ahuja N, Li Q, Mohan AL, Baylin SB, Issa JP. Aging and DNA methylation in colorectal mucosa and cancer. Cancer Res. 1998;58:5489-94.

20. Greco SA, Chia J, Inglis KJ, Cozzi SJ, Ramsnes I, Buttenshaw RL, et al. Thrombospondin-4 is a putative tumour-suppressor gene in colorectal cancer that exhibits age-related methylation. BMC Cancer. 2010;10:494.

21. Issa JP, Vertino PM, Boehm CD, Newsham IF, Baylin SB. Switch from monoallelic to biallelic human IGF2 promoter methylation during aging and carcinogenesis. Proc Natl Acad Sci U S A. 1996;93:11757-62.

22. Li Q, Jedlicka A, Ahuja N, Gibbons MC, Baylin SB, Burger PC, et al. Concordant methylation of the ER and N33 genes in glioblastoma multiforme. Oncogene. 1998;16:3197-202.

23. Florath I, Butterbach K, Müller H, Bewerunge-Hudler M, Brenner H. Crosssectional and longitudinal changes in DNA methylation with age: an epigenome-wide analysis revealing over 60 novel age-associated CpG sites. Hum Mol Genet. 2014;23:1186-201.

24. Bell JT, Tsai PC, Yang TP, Pidsley R, Nisbet J, Glass D, et al. Epigenome-wide scans identify differentially methylated regions for age and age-related phenotypes in a healthy ageing population. PLoS Genet. 2012;8:e1002629.

25. Cancer Genome Atlas Research Network. Comprehensive molecular profiling of lung adenocarcinoma. Nature. 2014;511:543-50.

26. Cancer Genome Atlas Research Network. Comprehensive genomic characterization of squamous cell lung cancers. Nature. 2012;489:519-25.

27. Um SW, Kim HK, Kim Y, Lee BB, Kim D, Han J, et al. Bronchial biopsy specimen as a surrogate for DNA methylation analysis in inoperable lung cancer. Clin Epigenetics. 2017;9:131

28. Slaughter DP, Southwick HW, Smehkal W. Field cancerization in oral stratified squamous epithelium; clinical implications of multicentric origin. Cancer. 1953; 6:963-8.

29. Kim DH, Nelson HH, Wiencke JK, Zheng S, Christiani DC, Wain JC, et al. p16 $6^{\mathrm{INK} 4 \mathrm{a}}$ and histology-specific methylation of $\mathrm{CpG}$ islands by exposure to tobacco smoke in non-small cell lung cancer. Cancer Res. 2001;61:3419-24.

30. Belinsky SA, Nikula KJ, Palmisano WA, Michels R, Saccomanno G, Gabrielson $E$, et al. Aberrant methylation of p16(INK4a) is an early event in lung cancer and a potential biomarker for early diagnosis. Proc Natl Acad Sci U S A. 1998:95:11891-6.

31. Zöchbauer-Müller S, Lam S, Toyooka S, Virmani AK, Toyooka KO, Seidl S, et al. Aberrant methylation of multiple genes in the upper aerodigestive tract epithelium of heavy smokers. Int J Cancer. 2003;107:612-6.
32. Buro-Auriemma LJ, Salit J, Hackett NR, Strulovici-Barel Y, Staudt MR, et al Cigarette smoking induces small airway epithelial epigenetic changes with corresponding modulation of gene expression. Hum Mol Genet. 2013;22: 4726-38.

33. Sood A, Petersen H, Blanchette CM, Meek P, Picchi MA, Belinsky SA, et al. Wood smoke exposure and gene promoter methylation are associated with increased risk for COPD in smokers. Am J Respir Crit Care Med. 2010;182: 1098-104.

34. Shimazu T, Asada K, Charvat H, Kusano C, Otake Y, Kakugawa Y, et al. Association of gastric cancer risk factors with DNA methylation levels in gastric mucosa of healthy Japanese: a cross-sectional study. Carcinogenesis. 2015;36:1291-8.

35. Maccani JZ, Koestler DC, Houseman EA, Marsit CJ, Kelsey KT. Placental DNA methylation alterations associated with maternal tobacco smoking at the RUNX3 gene are also associated with gestational age. Epigenomics. 2013;5: 619-30

36. Zhu X, Li J, Deng S, Yu K, Liu X, Deng Q, et al. Genome-wide analysis of DNA methylation and cigarette smoking in a Chinese population. Environ Health Perspect. 2016;124:966-73.

37. Zeilinger S, Kühnel B, Klopp N, Baurecht H, Kleinschmidt A, Gieger C, et al. Tobacco smoking leads to extensive genome-wide changes in DNA methylation. PLoS One. 2013;8:e63812.

38. Yanada M, Yaoi T, Shimada J, Sakakura C, Nishimura M, Ito K, et al. Frequent hemizygous deletion at 1p36 and hypermethylation downregulate RUNX3 expression in human lung cancer cell lines. Oncol Rep. 2005;14:817-22.

39. Sato K, Tomizawa $Y$, lijima H, Saito R, Ishizuka T, Nakajima T, et al. Epigenetic inactivation of the RUNX3 gene in lung cancer. Oncol Rep. 2006;15:129-35.

40. Subramaniam MM, Chan JY, Yeoh KG, Quek T, Ito K, Salto-Tellez M. Molecular pathology of RUNX3 in human carcinogenesis. Biochim Biophys Acta. 2009; 1796:315-31.

41. Lund AH, van Lohuizen M. RUNX: a trilogy of cancer genes. Cancer Cell. 2002;1:213-5.

42. Chen LF. Tumor suppressor function of RUNX3 in breast cancer. J Cell Biochem. 2012;113:1470-7.

43. Hwang JA, Lee BB, Kim Y, Park SE, Heo K, Hong SH, et al. HOXA11 hypermethylation is associated with progression of non-small cell lung cancer. Oncotarget. 2013:4:2317-25.

44. Selamat SA, Galler JS, Joshi AD, Fyfe MN, Campan M, Siegmund KD, Kerr KM Laird-Offringa IA, et al. DNA methylation changes in atypical adenomatous hyperplasia, adenocarcinoma in situ, and lung adenocarcinoma. PLoS One. 2011;6:e21443.

45. Anglim PP, Galler JS, Koss MN, Hagen JA, Turla S, Campan M, Weisenberger DJ, Laird PW, Siegmund KD, Laird-Offringa IA, et al. Identification of a panel of sensitive and specific DNA methylation markers for squamous cell lung cancer. Mol Cancer. 2008;7:62.

46. Rauch TA, Wang Z, Wu X, Kernstine KH, Riggs AD, Pfeifer GP. DNA methylation biomarkers for lung cancer. Tumour Biol. 2012;33:287-96.

47. Tessema M, Yingling CM, Grimes MJ, Thomas CL, Liu Y, Leng S, et al. Differential epigenetic regulation of TOX subfamily high mobility group box genes in lung and breast cancers. PLoS One. 2012:7:e34850.

\section{Ready to submit your research? Choose BMC and benefit from:}

- fast, convenient online submission

- thorough peer review by experienced researchers in your field

- rapid publication on acceptance

- support for research data, including large and complex data types

- gold Open Access which fosters wider collaboration and increased citations

- maximum visibility for your research: over $100 \mathrm{M}$ website views per year

At BMC, research is always in progress.

Learn more biomedcentral.com/submissions 